\title{
Coupled Electron Ion Monte Carlo Calculations of Dense Metallic Hydrogen
}

\author{
Carlo Pierleoni, ${ }^{1}$ David M. Ceperley, ${ }^{2}$ and Markus Holzmann ${ }^{3}$ \\ ${ }^{1}$ INFM and Department of Physics, University of L'Aquila, Via Vetoio, I-67010 L'Aquila, Italy \\ ${ }^{2}$ University of Illinois at Urbana-Champaign, Urbana, IL 61801, USA \\ ${ }^{3}$ LPTL, UMR 7600 of CNRS, Université P. et M. Curie, Paris, France
}

\begin{abstract}
We present a new Monte Carlo method which couples Path Integral for finite temperature protons with Quantum Monte Carlo for ground state electrons, and we apply it to metallic hydrogen for pressures beyond molecular dissociation. We report data for the equation of state for temperatures across the melting of the proton crystal. Our data exhibit more structure and higher melting temperatures of the proton crystal than Car-Parrinello Molecular Dynamics results. This method fills the gap between high temperature electron-proton Path Integral and ground state Diffusion Monte Carlo methods.
\end{abstract}

The knowledge of the physical properties of hydrogen in a wide range of thermodynamic conditions is a key problem in planetary and high pressure physics [1, 2]. In the search for the metallization transition three different insulating molecular crystal phases have been clearly observed so far in diamond anvil cell experiments up to $3.2 \mathrm{Mbar}[3$ at room temperature and below. Metallization has been obtained in shock wave experiments for a warm dense molecular liquid [4] but properties at finite temperature and/or at higher pressure are largely unknown because experiments are increasingly difficult.

A large body of theoretical investigations of high pressure hydrogen have appeared over the years [5]. They helped the understanding of the experimental observations and hold out the prospect of predicting the room temperature metallization pressure and the phase diagram at higher pressure. However the present understanding of high pressure hydrogen is unsatisfactory because 1) energy differences among different crystalline phases are small requiring a very accurate total energy method to determine the stable crystalline phase and locate transition lines; 2) size effects are large in metallic and quasi-metallic systems and Brillouin zone sampling is extremely important for accurate total energy calculations; 3) proton quantum effects are important and can influence the energetic ordering of crystal phases ; 4) an accurate theoretical prediction of metallization may require accuracy beyond that of the LDA+GGA Density Functional Theory [6, 7].

Here we describe a method based on Quantum Monte Carlo (QMC) calculation of the electronic energy for quantum mechanical protons able to sample efficiently the protonic configurational space and spontaneously find the stable phase of the system within the BornOppenheimer approximation. Previous QMC studies of hydrogen at $T=0$ have treated electrons and protons at the same level of description and become inefficient in following the evolution of particles of very dissimilar mass $\left(m_{p} / m_{e}=1836\right)$. Moreover, the interesting effects of temperature are absent in this procedure. Nonetheless, they have established that pressure dissociation of hydrogen molecules at $\mathrm{T}=0 \mathrm{~K}$ occurs at $r_{s}=\left[3 /\left(4 \pi n_{e}\right)\right]^{1 / 3}=1.31(P \sim 3$ Mbars $)[\underline{8}]$, where $n_{e}$ is the electronic number density. Upon dissociation the molecular crystal transforms to a proton lattice of diamond structure and later to a lattice of cubic symmetry (bcc) at $P \geq 8$ Mbars 9 , 10. At finite temperature Restricted Path Integral Monte Carlo (RPIMC) 11 has been used to predict the equation of state (EOS) and to investigate the occurrence of the plasma phase transition 12. In RPIMC, both electrons and protons are at finite temperature but it is efficient only for temperatures above $1 / 20$ of the electronic Fermi temperature (roughly $3 \times 10^{4} \mathrm{~K}$ at $r_{s}=1$ ). The new method described here, called Coupled Electronic-Ionic Monte Carlo (CEIMC) 13, 14], is able to fill the gap between the RPIMC and the ground state QMC methods. We study metallic hydrogen in a range of densities and temperatures where molecules are absent and where protons undergo a solid-fluid transition. We report results for the EOS and give a qualitative location of the transition line.

In the CEIMC method the proton degrees of freedom are advanced by a Metropolis algorithm in which the energy difference between the actual state $S$ and the trial state $S^{\prime}$ is computed by a Quantum Monte Carlo calculation (either variational (VMC) or reptation (RQMC) 15]). The energy difference is affected by statistical noise which would bias the MC sampling. Unbiased sampling of the proton configurations can be achieved by the penalty method [16], a generalization of the Metropolis algorithm.

We sample the electronic degrees of freedom according to the sum of the electronic distribution functions (e. $g$. the square of the trial wave function in VMC) for the $S$ and $S^{\prime}$ states, and we compute the energies for the two states as correlated sampling averages 13, 14], thereby reducing the noise. Analytic trial wave functions including backflow and three-body correlation 17 . have been used in most of our calculations. These functions are particularly appropriate to our methods since: 1) they are quite accurate; 2 ) they are free of adjustable parameters so do not require optimization; 3) their computational cost is much less then solving the Kohn-Sham equations as was done in previous QMC calculations 9,10 , in particular 
for a random arrangement of several tens of protons.

To go beyond VMC, we implemented a Reptation Quantum Monte Carlo algorithm (RQMC) 15. to sample more accurately the electronic ground state. Similar to Diffusion Monte Carlo (DMC), RQMC projects the trial wavefunction to the ground state within the Fixed-Node approximation. The high quality of our trial wave functions makes it possible to relax to the ground state with a very limited number of time slices. In RQMC the electronic path space is sampled by a reptation algorithm in which, at each step, a new link is added to one end of the path and an existing link is deleted from the other end, subject to a "Metropolis" acceptance/rejection step. To speed up convergence we have introduced the "bounce" algorithm in which the growth direction is reversed only when a move is rejected 18. RQMC is particularly suited for computing energy differences.

To reduce finite size effects in metallic systems, we average over twisted boundary conditions (TABC) when computing electronic energies (i.e. we integrate over the Brillouin zone of the super cell) 14, 19]. All properties are averaged over 1000 different k-points. For the typical protonic displacement, we compute the energy difference over 100 electronic steps/k-point. After averaging over $\mathrm{k}$-points, the noise level is small enough to simulate temperatures as low as $100 \mathrm{~K}[14]$.

We represent protons by imaginary time path integrals without considering the statistics of the protons. (those effects are negligible in this temperature-density range.) For efficiency, it is important to minimize the number of protonic time slices. We have used the pair action of an effective proton-proton potential and treated the difference between the true Born-Oppenheimer energy and the effective potential with the primitive approximation [20]. With this action, we find that a proton imaginary time step $\tau_{p}=0.3 \times 10^{-3} K^{-1}$ is appropriate for $r_{s} \geq 1$ so that few tens of time slices allow for calculations above $100 \mathrm{~K}$. When coupled with TABC, we can, at each proton move, randomly assign a subset of k-points to each protonic slice without introducing a detectable systematic effect. This strategy allows one to simulate quantum protons at essentially the same computational cost as classical protons, except for the slower relaxation of the protonic paths.

In order to assess the accuracy of the CEIMC method we first consider a system of $N_{p}=N_{e}=16$ at $r_{s}=1$ and $\mathrm{T}=5000 \mathrm{~K}$ and compare with RPIMC. The CEIMC-RQMC calculation, performed with $\tau_{e}=$ $0.0125 H^{-1}, \beta_{e}=0.5 H^{-1}$ (41 electronic time slices), provides a total energy lower than the VMC estimate by $4(2) \mathrm{mH} /$ atom $=1260(630) \mathrm{K}$.However, the $\mathrm{VMC}$ and RQMC pressures agree within error bars. Comparison between VMC and RQMC pair correlation functions is also very good (see figure (1). The VMC and RQMC $g_{e p}(r)$ 's are superimposed except at distance below $0.2 a_{0}$ ( $a_{0}=0.529 \AA$ is the Bohr radius), due to time step er-

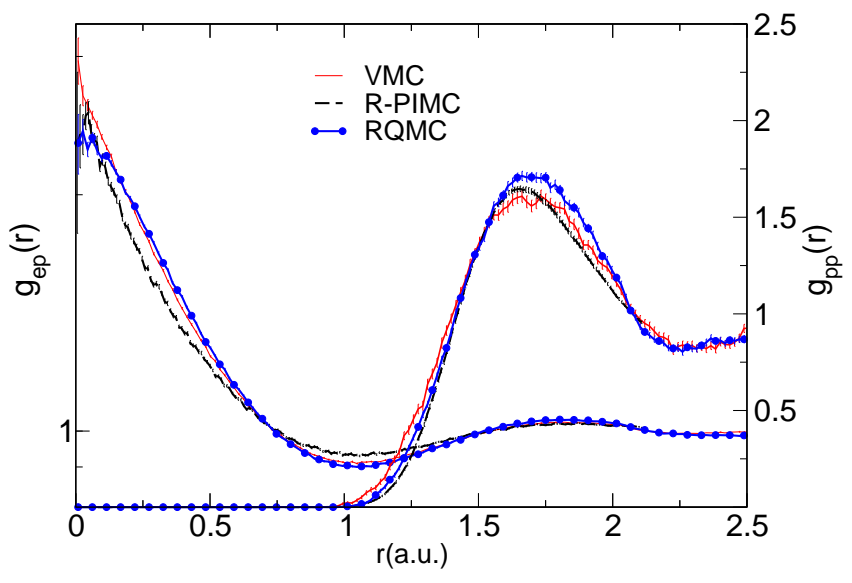

FIG. 1: CEIMC-RPIMC comparison for electron-proton and proton-proton correlation function at $r_{s}=1, T=$ $5000 K, N_{p}=N_{e}=16$.

ror in RQMC. As for $g_{p p}(r)$, RQMC curve is slightly more structured than the VMC one. In RPIMC, such "low" temperatures (the Fermi temperature at $r_{s}=1$ is $1.84 \mathrm{H}=5.810^{5} \mathrm{~K}$ ) can be reached only by imposing less realistic ground state nodal restriction 11, 14]. RPIMC data, obtained with free particle nodes and 1000 time slices, agrees with CEIMC ones. CEIMC computed $g_{e p}(r)$ exhibits slightly more structure than RPIMC, and since thermal effects on the electrons should be largely negligible in such conditions, we attribute the observed difference to the more accurate nodal structure of CEIMC compared to RPIMC.

Next we compare with Car-Parrinello Molecular Dynamics (CPMD) simulation 21] which uses the LDA computed forces. Figure 2 shows that CEIMC-VMC $g_{p p}(r)$ 's for classical protons exhibit considerably more structure than does LDA. CPMD simulations considered systems of classical protons with a closed shell (in reciprocal space), and only the $\Gamma$ point. We compare with two different CEIMC calculations for classical protons, namely an open shell system $\left(N_{p}=32\right)$ with the TABC, and a closed shell system $\left(N_{p}=54\right)$ with the $\Gamma$ point only. For the latter case, we find that the $g_{p p}(r)$ from VMC and RQMC (not shown) agree; but they exhibit more structure than CPMD. The TABC one is in the liquid state, while the simulation using only the $\Gamma$ point, initially prepared in a liquid state from temperature quenching, exhibits the onset of spontaneous crystallization. The larger correlation in CEIMC with respect to CPMD is compatible with our early estimate of the melting temperature of the fcc crystal of classical proton between $1000 \mathrm{~K}$ and $1500 \mathrm{~K}$ 14] at variance with the the LDA estimate of $350 \mathrm{~K}$ (for the bcc crystal) 21]. The observed discrepancy between CEIMC and CPMD is surprising since LDA is generally believed to be accurate at high density. However a previous study of hydrogen at $r_{s}=1.31$ [ ] reported that differences in energy among several crys- 


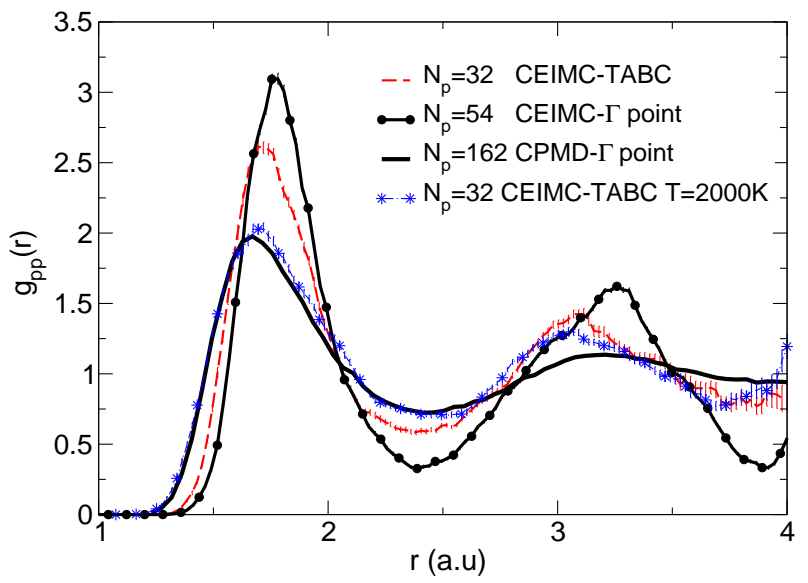

FIG. 2: Pair correlation function at $r_{s}=1, T=1000 \mathrm{~K}$. Comparison between CEIMC-VMC-TABC with $N_{p}=32$, CEIMC-VMC-PBC $N_{p}=54 \mathrm{~s}$ and CPMD-LDA $N_{p}=162$ (simulation with $N_{p}=54$ is identical). Data from CEIMCVMC-TABC at $\mathrm{T}=2000 \mathrm{~K}$ (stars) are also reported.

tal structures obtained within LDA are smaller than energy differences from Diffusion MC by roughly a factor of two. Also zero point energies in QMC were roughly twice the LDA estimates (from the harmonic approximation). This suggests that the Born-Oppenheimer surface from LDA is flatter than the more accurate one from QMC. Moreover there is a known issue in computing the ionic temperature in CPMD; the simple estimate based on the ionic kinetic energy provides only a lower bound for the true temperature 22]. Tracing the origin of the observed discrepancy between CEIMC and CPMD results would deserve an independent study. Here we just note that better agreement is observed between CPMD results at temperature $\mathrm{T}$ and CEIMC data at temperature $2 \mathrm{~T}$ for $300 \leq T \leq 3000$, see for instance figure 2

RQMC is roughly an order of magnitude more expensive than VMC. Therefore, it is important to establish the accuracy of VMC before performing a systematic study of the equation of state. In table 【 we compare $\mathrm{VMC}$ and RQMC at $T=5000 K$ and $r_{s}=1.2$, the lowest density we have considered. Calculations are done at the $\Gamma$ point and a projection time of $\beta_{e}=0.68 \mathrm{H}^{-1}$ for RQMC. We have checked that for protons in bcc lattice, the energy has converged to its ground state value for $\beta \sim 0.6$. We find that the VMC energy is systematically higher by roughly $7.6(2) \mathrm{mH} / \mathrm{at}=2400(60) \mathrm{K}$, while the VMC pressure is systematically lower by 0.03(1)Mbars. The error on the energy is expected to be independent of the temperature and to decrease with increasing density. Even though the amount of energy missing in VMC is quite large on the proton energy scale, we only observe a minor effect on $g_{p p}(r)$; energy differences are quite accurate within VMC. On the basis of the above results, we performed a systematic study of the Equation of State (EOS) using VMC. In table III we report
TABLE I: Comparison between VMC and RQMC energies and pressures for $r_{s}=1.2, T=5000 K, N_{p}=N_{e}=54, \mathrm{PBC}$. $\tau=0$ is the estimate with RQMC time step errors removed by extrapolation. $\sigma^{2}$ is the variance of the local energy in VMC.

\begin{tabular}{|c|ccccc|}
\hline$\tau_{e}$ & $\mathrm{E}_{\text {tot }}(\mathrm{h} / \mathrm{at})$ & $\sigma^{2}$ & $\mathrm{E}_{k i n}$ & $\mathrm{E}_{\text {pot }}$ & $\mathrm{P}$ (Mbars) \\
\hline $\mathrm{vmc}$ & $-0.4694(2)$ & $0.0472(4)$ & $0.8812(4)$ & $-1.3508(4)$ & $5.55(1)$ \\
0.01 & $-0.4768(4)$ & - & $0.8850(6)$ & $-1.3618(6)$ & $5.50(1)$ \\
0.00 & -0.47696 & - & 0.89112 & -1.36808 & 5.581 \\
\hline
\end{tabular}

total, kinetic and potential energies, pressure, the Lindemann ratio for bcc crystal, and the proton kinetic energy. The latter quantity can be compared to $3 K_{B} T / 2$ (last column). The zero point proton motion affects not only the proton kinetic energy but also increases the electronic kinetic energy and, to a smaller extent, the configurational energy. At $r_{s}=1$ and $T=500 \mathrm{~K}$ we find a total energy increase of $14.9(2) \mathrm{mH} / \mathrm{at}=4670(60) \mathrm{K}$ of which $2020(30) K$ comes from the proton kinetic energy, $2200(20) K$ the electronic kinetic energy, and 450(10) $K$ the configurational energy. Residual finite size effects have been estimated from static lattice calculation at $r_{s}=1$ to be of the order of $10 \mathrm{mH} / \mathrm{at}$ on the energy, and 0.21 Mbars on the pressure. The transition line, estimated by the dynamical observation of melting, is located between $1000 \mathrm{~K}$ and $2000 \mathrm{~K}$ at $r_{s}=0.8$, between $500 \mathrm{~K}$ and $1000 \mathrm{~K}$ at $r_{s}=1.0$ and close to $1000 \mathrm{~K}$ at $r_{s}=1.2$. Indeed at the latter density and at $\mathrm{T}=1000 \mathrm{~K}$ the system is able to sustain both liquid and crystal states for the entire length of our simulations (80000 protonic steps).

In conclusion we have developed a new and efficient Quantum Monte Carlo Method to study low temperature quantum protons and ground state electrons which is a major improvement over previous QMC and DFT-LDA based methods. It allows for simulations of many-body hydrogen using QMC for the electronic energies. We have developed efficient procedures to include protonic path integrals and k-point sampling. We have applied it to metallic hydrogen beyond molecular dissociation and investigated the solid-fluid transition of the protons. The present methodology can be extended in several ways. Constant pressure algorithm would be useful to study structural phase transitions. However for metallic systems, we have found that level crossings, arising from changes in the shape of the simulation box, considerably increase the noise level and makes our correlated sampling procedure inefficient. The method can be easily extended to the insulating molecular phase by replacing the metallic trial functions with localized molecular orbitals 13, 14]. A study of the melting line of molecular hydrogen is in progress. Consideration of the metalinsulator transition requires a trial function that goes smoothly from metallic to localized orbitals. We are investigating an accurate and efficient form for this. Extension of the present method to more complex elements 
TABLE II: Energy and pressure for a system of $N_{p}=54$ quantum protons with VMC-TABC. Units of energy are hartrees/proton. $M_{p}$ is the number of protonic time slices ( $M_{p}=1$ means classical protons). $\gamma$ is the rms deviation divided by the nearest neighbor distance for a bcc lattice.

\begin{tabular}{|c|c|c|ccc|c|c|cc|}
\hline$r_{s}$ & $\mathrm{~T}(\mathrm{KK})$ & $M_{p}$ & $E$ & $E_{k i n}$ & $E_{\text {pot }}$ & $P($ Mbars $)$ & $\gamma_{L}$ & $K_{p} \times 10^{2}$ & $K_{p}^{c l} \times 10^{2}$ \\
\hline 0.8 & 0.5 & 16 & $-0.0594(2)$ & $1.8419(1)$ & $-1.9033(1)$ & $81.07(3)$ & $0.169(1)$ & $1.57(3)$ & 0.2375 \\
& 1.0 & 16 & $-0.0586(4)$ & $1.8428(4)$ & $-1.9034(1)$ & $81.16(3)$ & $0.183(1)$ & $1.53(4)$ & 0.475 \\
& 2.0 & 8 & $-0.0522(4)$ & $1.8338(4)$ & $-1.9018(1)$ & $81.69(3)$ & - & $1.78(3)$ & 0.950 \\
& 3.0 & 4 & $-0.0442(4)$ & $1.8538(6)$ & $-1.9000(2)$ & $82.33(6)$ & - & $2.14(7)$ & 1.425 \\
& 4.0 & 4 & $-0.0382(8)$ & $1.8590(8)$ & $-1.8991(1)$ & $82.83(6)$ & - & $2.57(7)$ & 1.900 \\
& 6.0 & 2 & $-0.0268(8)$ & $1.8688(8)$ & $-1.8974(2)$ & $83.80(6)$ & - & $3.29(4)$ & 2.850 \\
& 10.0 & 1 & $0.016(1)$ & $1.8886(8)$ & $-1.8934(4)$ & $85.78(9)$ & - & 4.750 & 4.750 \\
\hline 1.0 & 0.5 & 8 & $-0.3512(2)$ & $1.2142(2)$ & $-1.5655(1)$ & $20.101(3)$ & $0.177(1)$ & $0.97(2)$ & 0.2375 \\
& 1.0 & 4 & $-0.3480(2)$ & $1.2176(2)$ & $-1.5657(1)$ & $19.68(1)$ & - & $1.07(2)$ & 0.475 \\
& 2.0 & 4 & $-0.3430(2)$ & $1.2260(4)$ & $-1.5653(1)$ & $20.65(1)$ & - & $1.44(2)$ & 0.950 \\
& 3.0 & 2 & $-0.3356(4)$ & $1.2298(4)$ & $-1.5655(1)$ & $20.83(1)$ & - & $1.72(3)$ & 1.425 \\
& 5.0 & 1 & $-0.3262(6)$ & $1.2390(6)$ & $-1.5652(1)$ & $21.26(2)$ & - & 2.375 & 2.375 \\
& 10.0 & 1 & $-0.2888(6)$ & $1.2740(4)$ & $-1.5630(2)$ & $22.95(3)$ & - & 4.750 & 4.750 \\
\hline 1.2 & 0.3 & 10 & $-0.46610(4)$ & $0.8776(1)$ & $-1.3437(1)$ & $5.554(1)$ & $0.134(1)$ & $0.59(1)$ & 0.1425 \\
& 0.5 & 8 & $-0.4661(1)$ & $0.8792(1)$ & $-1.3439(1)$ & $5.594(3)$ & $0.177(2)$ & $0.67(1)$ & 0.2375 \\
& 1.0 & 4 & $-0.4632(1)$ & $0.8811(2)$ & $-1.3443(2)$ & $5.641(3)$ & $0.196(3)$ & $0.77(1)$ & 0.475 \\
& 1.0 & 4 & $-0.4610(2)$ & $0.8858(2)$ & $-1.3468(1)$ & $5.735(6)$ & 1 iquid & $0.77(1)$ & 0.475 \\
& 2.0 & 4 & $-0.4552(2)$ & $0.8918(2)$ & $-1.3469(1)$ & $5.893(6)$ & - & $1.19(3)$ & 0.950 \\
& 3.0 & 2 & $-0.4492(4)$ & $0.8996(3)$ & $-1.3488(1)$ & $6.08(2)$ & - & $1.53(3)$ & 1.425 \\
& 5.0 & 1 & $-0.4386(6)$ & $0.9106(4)$ & $-1.3492(2)$ & $6.37(2)$ & - & 2.375 & 2.375 \\
& 10.0 & 1 & $-0.4036(6)$ & $0.9478(4)$ & $-1.3514(1)$ & $7.34(2)$ & - & 4.750 & 4.750 \\
\hline
\end{tabular}

is straightforward, provided we have efficient trial functions.

Early aspects of the CEIMC algorithm were developed in collaboration with M. Dewing. We have the pleasure to thank J.P.Hansen and J.Kohanoff for useful discussions and for providing their CPMD data, and S.Scandolo and R.M. Martin for illuminating discussions. This work has been supported by a visiting grant from INFM-SezG and by MIUR-COFIN-2003. Computer time has been provided by NCSA (Illinois), PSC (Pennsylvania) and CINECA (Italy) through the INFM Parallel Computing initiative.

[1] D.J. Stevenson in High Pressure Phenomena, ed. by R.J.Hemley et al. (IOS Press, Amsterdam 2002)

[2] H. Mao and R.J. Hemley, Rev. Mod. Phys. 66, 671 (1994)

[3] P. Luobeyre, F. Occelli and R. Le Toullec, Nature 416, 613 (2002).

[4] W.J.Nellis, M. Ross and N.C. Holmes, Science 269, 1249 (1995); S.T.Weir, A.C. Mitchell and W.J. Nellis, Phys. Rev. Lett. 76, 1860 (1996).

[5] E.G.Maksimov and Yu I.Silov, Physics-Uspekhi, 42, 1121 (1999).

[6] M. Stadele and R.M. Martin, Phys. Rev. Lett. 84, 6070 (2000)

[7] K.A.Johnson and N.W.Ashcroft, Nature 403, 632 (2000)

[8] D.M. Ceperley and B.J. Alder, Physica 108B, 875 (1981); Phys. Rev. B36, 2092 (1987).

[9] V. Natoli, R. M. Martin, and D. M. Ceperley, Phys. Rev.
Lett. 70, 1952 (1993).

[10] V. Natoli, R. M. Martin and D. Ceperley, Phys. Rev. Lett. 74, 1601 (1995).

[11] D. M. Ceperley in Monte Carlo and Molecular Dynamics of Condensed Matter Systems, ed. by K. Binder and G. Ciccotti (Editrice Compositori, Bologna, Italy, 1996).

[12] C. Pierleoni, D. M. Ceperley, B. Bernu and W. R. Magro: Phys. Rev. Lett. 73, 2145 (1994); W. R. Magro, D. M. Ceperley, C. Pierleoni, and B. Bernu: Phys. Rev. Lett. 76, 1240 (1996); B. Militzer and D. M. Ceperley: Phys. Rev. Lett. 85, 1890 (2000).

[13] M. Dewing and D. M. Ceperley, in Recent Advances in Quantum Monte Carlo Methods, II, ed. S. Rothstein, World Scientific (2002).

[14] D. M. Ceperley, M. Dewing and C. Pierleoni. Lecture Notes in Physics "Bridging Time Scales", Vol 605, pg. 473-499. ed. P. Nielaba et al., Springer-Verlag (2003), physics/0207006

[15] S. Baroni and S. Moroni, Phys. Rev. Lett 82, 4745 (1999).

[16] D. M. Ceperley and M. Dewing: J. Chem. Phys. 110, 9812 (1999)

[17] M. Holzmann, D. M. Ceperley, C. Pierleoni and K. Esler, Phys. Rev E 68, 046707 (2003).

[18] D.M. Ceperley and C. Pierleoni, to be published.

[19] C. Lin, F. H. Zong and D. M. Ceperley, Phys. Rev. E 64, 016702 (2001).

[20] D. M. Ceperley, Rev. Mod. Phys. 67, 279 (1995).

[21] J.Kohanoff and J.P.Hansen, Phys. Rev. Lett. 74, 626 (1995); Phys. Rev. E 54, 768 (1996).

[22] P. Tangney and S. Scandolo, J. Chem. Phys. 116, 14 (2002). 